\title{
A method of the forward problem for magneto-acousto-electrical tomography
}

\author{
Jingxiang Lv $v^{\mathrm{a}, \mathrm{b}, \mathrm{c}}$, Guoqiang Liu ${ }^{\mathrm{a}, *}$, Xinli Wang ${ }^{\mathrm{a}}$ and Hui Xia ${ }^{\mathrm{a}}$ \\ ${ }^{a}$ Institute of Electrical Engineering, Chinese Academy of Sciences, Beijing, China \\ ${ }^{\mathrm{b}}$ University of Chinese Academy of Sciences, Beijing, China \\ ${ }^{\mathrm{c}}$ School of Electronics and Information, Jinggangshan University, Ji'an, Jiangxi, China
}

\begin{abstract}
Magneto-Acousto-Electrical Tomography (MAET) is a novel hybrid modality that can provide a high spatial resolution in determining the electrical conductivity of biological tissue. The present paper primarily analyzes the existing basic formulations with the MAET, derives the propagation equations of the sound wave when the mass density of the biological tissues are variable, and then solves the respective current density and potential difference in an inhomogeneous and homogeneous density medium based on the sound speeds obtained. Finally, numerical simulations are performed. As is shown, sound waves affect magneto-acousto-electrical tomography while varying the biological tissue mass density.
\end{abstract}

Keywords: Acoustic characteristic, forward problem, MAET, conductivity

\section{Introduction}

Ultrasonic imaging $[1,2]$ is widely utilized in medical imaging due to its high spatial resolution and relatively low cost. However, it is difficult to differentiate between soft tissues because the acoustic impedance in muscle and fat is nearly identical. The electrical conductivity of the biological tissue potentially has a high contrast; accordingly, this has garnered significant attention from medical imaging researchers. Information about the pathological and physiological condition of tissues can be obtained by measuring the electrical conductivity. A popular technique for measuring the electrical conductivity is electrical impedance tomography. However, this method is limited by an inverse problem and lower spatial resolution [3,4].

At present, several approaches have been developed to improve the spatial resolution of electrical impedance tomography, including magnetic resonance electrical impedance tomography (MREIT) [5], magneto acoustic tomography (MAT) [6], magneto acoustic current imaging, and magneto-acoustoelectrical tomography (MAET). Magneto-acousto-electrical tomography techniques have the potential for high spatial resolution; thus, this technology overcomes the low spatial resolution of electrical impedance tomography.

Magneto-acousto-electrical tomography integrates the advantages of both traditional EIT and ultrasonic imaging. In the MAET method [10], an ultrasound pulse propagates through the biological tissue

\footnotetext{
${ }^{*}$ Corresponding author: Guoqiang Liu, Institute of Electrical Engineering, Chinese Academy of Sciences, Beijing 100190, China. E-mail: gqliu@mail.iee.ac.cn.
}

0928-7329/16/\$35.00 @ 2016 - IOS Press and the authors. All rights reserved

This article is published online with Open Access and distributed under the terms of the Creative Commons Attribution NonCommercial License. 
to be imaged in the presence of a static magnetic field. The ultrasonic wave induces Lorentz force on the ions in the biological tissue, which causes the positively and negatively charged ions to separate. This separation of charge generates a local source of electrical current or voltage. Therefore, there will be a corresponding electric field distribution in biological tissues. From the voltage data measured by electrodes on the surface of biological tissues, the conductivity distribution can be reconstructed by adopting a reconstruction algorithm. For example, the Compression Sensing method can be introduced into the reconstruction of the conductivity distribution. In order to solve the ill-posed character, the damping least square algorithm was used to reconstruct the conductivity.

Various studies have been conducted on the development and improvement of the MAET technique. Wen et al. developed the MAET known as the Hall effect imaging [7]. Kunyansky researched the inversion procedure for magneto-acousto-electrical tomography [8]. Additionally, Roth developed various methods for MAET as well as indicated several limitations of the developed methods [9].

At present, magneto-acousto-electrical tomography has primarily been used to investigate homogenous biological tissues. However, in a variety of MAET applications, heterogeneous biological tissues are unavoidable. For example, in [11], a study was conducted on the electrical and acoustic properties of biological materials, and biomedical applications were explored. The acoustic wave propagation in the inhomogeneous medium has attracted the interests of numerous researchers. In this paper, the propagation equation of the acoustic wave that propagates in variable mass density is derived.

\section{Basic formulations of MAET}

\subsection{Forward problem of the electromagnetic field}

Suppose that an object of interest is reconstructed with the electrical conductivity $\sigma$, the bounded region $\Omega$, and the boundary $\partial \Omega$. The object is placed in the magnetic field with a constant magnetic induction $B$, through which an acoustic wave propagates with the velocity $v$. The produced Lorentz force will generate the location current source given by the following formula:

$$
J_{s}=\sigma v \times B
$$

In addition to the local current source, the arising electrical potential $u$ generates the secondary Ohmic currents $J_{0}$ according to Ohm's law.

$$
J_{0}=-\sigma \nabla u
$$

Since there are no sinks or sources of charges in the object of interest, the total current density is divergence free.

$$
\nabla \cdot\left(J_{s}+J_{0}\right)=0
$$

Thus,

$$
\nabla \cdot \sigma \nabla u=\nabla \cdot(\sigma v \times B)
$$

Since there are no currents through the boundary, the normal component of the total current vanishes. It is shown as follows:

$$
\left.\frac{\partial u}{\partial n}\right|_{\partial \Omega}=(v \times B) \cdot n
$$

In Eq. (5) given above, $n$ is the exterior normal to $\partial \Omega$.

It is necessary to first obtain $v$ using the sound wave equation so that $u$ can be solved. Then, a method that sound wave propagation in non-uniform and unsteady acoustic medium must obey is shown as follows. 


\subsection{The acoustic wave equation to inhomogeneous medium}

In present MAET research, the acoustic properties of biological tissue are normally treated as the constant media. In this case, the acoustic pressure satisfies the following wave equation in $\Omega$ :

$$
\frac{1}{c_{0}^{2}} \frac{\partial^{2} p}{\partial t^{2}}=\nabla^{2} p
$$

However, it is clear that in some cases, the speed of sound and the density of the tissues cannot generally be regarded as constant. In this paper, the sound wave equation is derived when the sound speeds and the densities of the tissues are variable.

Acoustic kinetic per unit volume for sound potential is represented as:

$$
E_{v}=\frac{1}{2} \rho_{0} v^{2}=\frac{1}{2} \rho_{0}(\nabla \phi)^{2}
$$

The relationship between the acoustic pressure and the sound potential in incompressible fluid is that the acoustic pressure is the time derivative of the velocity potential as shown in Eq. (8):

$$
p=-\rho_{0} \frac{d \phi}{d t}
$$

Potential energy is written as:

$$
E_{p}=\frac{p^{2}}{2 \rho_{0} c_{0}^{2}}=\frac{1}{2}\left(\frac{\rho_{0}}{c_{0}^{2}}\right)\left(\frac{d \phi}{d t}\right)^{2}
$$

The Acoustic Lagrange function is the difference between the kinetic energy and potential energy.

$$
\begin{aligned}
& L(\phi ; \dot{\phi}, \nabla \varphi ; r, t)=E_{v}-E_{p} \\
& \quad=\frac{1}{2} \rho_{0}\left[(\nabla \phi)^{2}-c_{0}^{-2}\left(\frac{d \phi}{d t}\right)^{2}\right]
\end{aligned}
$$

The Euler-Lagrange equation of non-uniform and unsteady acoustic medium can be written as:

$$
\frac{\partial(\partial L / \partial \dot{\phi})}{\partial t}+\nabla \cdot\left[\frac{\partial L}{\partial(\nabla \phi)}\right]=0
$$

Because the biological tissue in medical imaging is stationary, the acoustic function of Lagrange can be simplified as:

$$
L=\frac{1}{2} \rho_{0}\left[(\nabla \phi)^{2}-c_{0}^{-2} \dot{\phi}^{2}\right]
$$

The equation $\partial \rho_{0} / \partial t=0$ holds in the static organization, so the following can be derived when Eq. (12) is substituted in Eq. (11):

$$
\frac{\partial\left(c_{0}^{-2} \dot{\phi}\right)}{\partial t}-\rho_{0}^{-1} \nabla \cdot\left(\rho_{0} \nabla \phi\right)=0
$$

In this paper, $c_{0}$ is considered constant, so the sound potential in the biological tissue satisfies the following equation:

$$
c_{0}^{-2} \ddot{\phi}-\rho_{0}^{-1} \nabla \cdot\left(\rho_{0} \nabla \phi\right)=0
$$




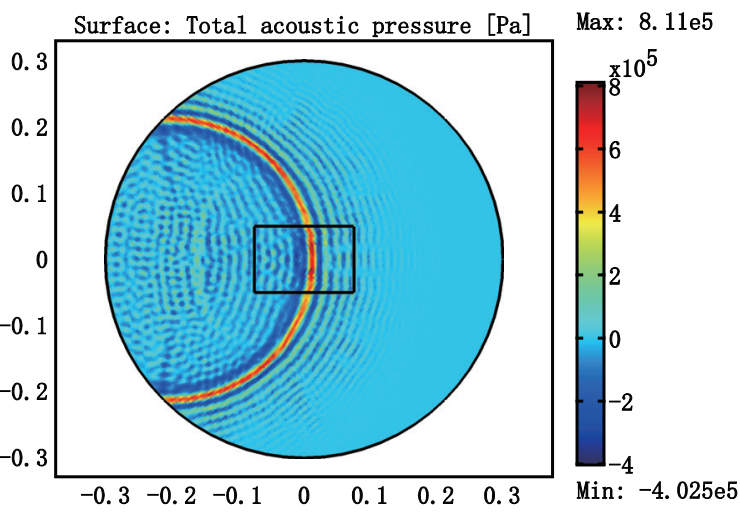

Fig. 1. Schematic diagram of acoustic pressure in the constant mass densities.

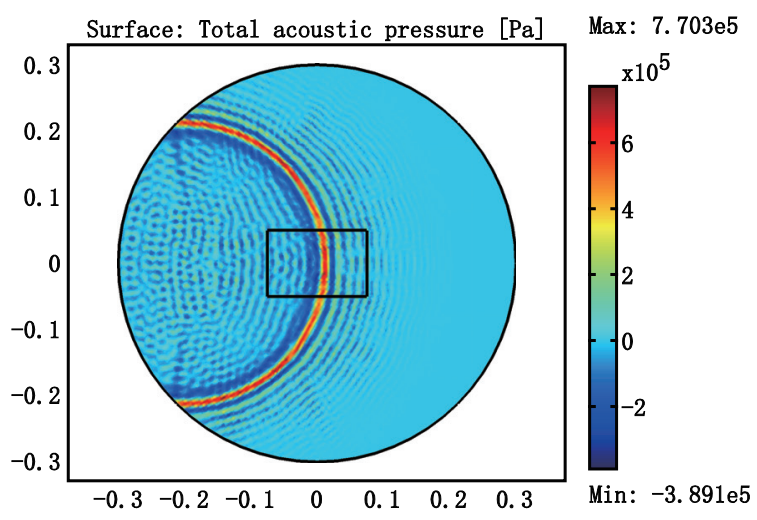

Fig. 2. Schematic diagram of acoustic pressure in the variable mass densities.

When Eq. (8) is substituted in Eq. (14), the wave equation that the acoustic pressure satisfies in $\Omega$ can be derived. The vibration velocity can be obtained by Eq. (16).

$$
\begin{aligned}
& \left\{\begin{array}{l}
1 /\left(\rho_{0} c_{s}^{2}\right) \partial^{2} p / \partial t^{2}+\nabla \cdot\left(-\left(1 / \rho_{0}\right) \nabla p\right)=0 \\
\left.p\right|_{t=0^{-}}=0 \\
\frac{\partial p}{\partial t} t=0^{-} \\
n \cdot \nabla p=0
\end{array}\right. \\
& \rho_{0} \frac{\partial v}{\partial t}=-\nabla p / \partial t \quad \text { in boundary }
\end{aligned}
$$

\section{Numerical simulation results}

This algorithm is verified by a two-step procedure: (1) the acoustic pressure $p$ is calculated numerically in the tissue when the densities of the tissues are approximately constant and variable, respectively, and (2) the surface current density distribution and potential difference are calculated numerically by taking into account the acoustic field coupled with the electromagnetic field.

A 2D model is built to simulate the ultrasound pulse propagation in a sample. The tissue is assumed to be rectangular with a width of $20 \mathrm{~mm}$ and a height of $10 \mathrm{~mm}$, and the outer boundary of the tissue is circular and satisfies infinite boundary conditions. Further, the sound source is assumed to obey a Gaussian distribution, and the sound pressure peaks at $1 \mathrm{MPa}$ propagating along $\mathrm{x}$. The mass density of the sample is $1000 \mathrm{~kg} / \mathrm{m}^{3}$, and the electrical conductivity is $1 \mathrm{~s} / \mathrm{m}$. The sound speed is $1500 \mathrm{~m} / \mathrm{s}$, and the sample is steeped in water that has a density and sound speed that are identical to the tissue. The distance between the ultrasound transducer and the sample is $12.5 \mathrm{~mm}$. In Figs 1 and 3, the acoustic pressure and the surface current density in which the mass density and the electrical conductivity are constant are respectively shown. In contrast, the density of the biological tissue is changed, but the electrical conductivity remains $1 \mathrm{~s} / \mathrm{m}$. For example, a Gaussian distribution for the mass densities is considered. In general, the tissue densities remain within about $1100 \mathrm{~kg} / \mathrm{m}^{3}$ except for human bones; thus, the variable density is $1100 *\left(\exp \left(-\left(x^{\wedge} 2+y^{\wedge} 2\right) / 0.03 * 0.03\right)\right)$ [8]. The acoustic pressure and the surface current density are shown respectively in Figs 2 and 4. In MAET, the potential difference measured in the surface is conducive to the reconstruction of the electrical conductivity of the tissues, and as a result, it is stimulated as shown in Fig. 5. 


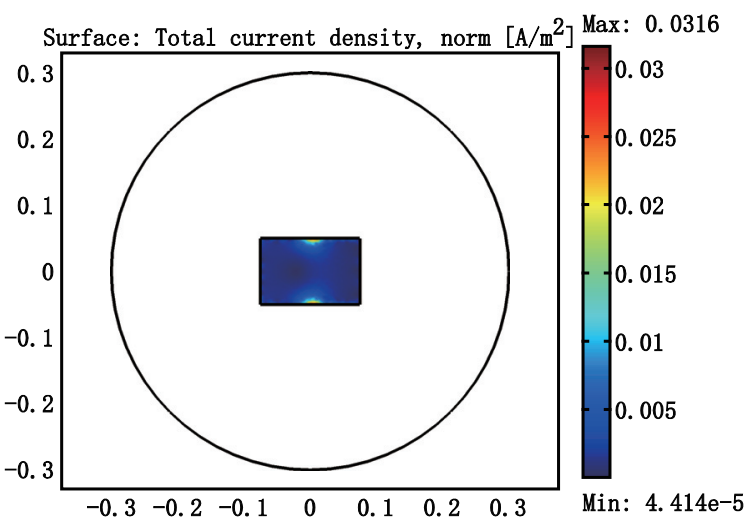

Fig. 3. Schematic diagram of the surface current density in the constant mass densities.

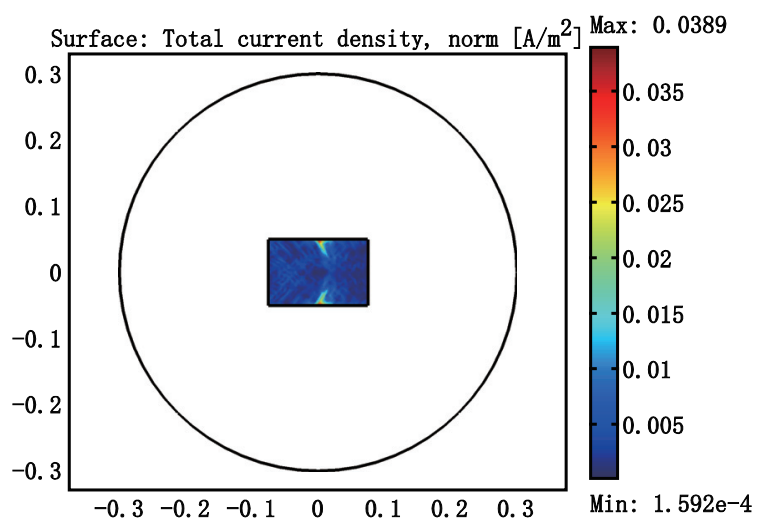

Fig. 4. Schematic diagram of the surface current density in the variable mass densities.

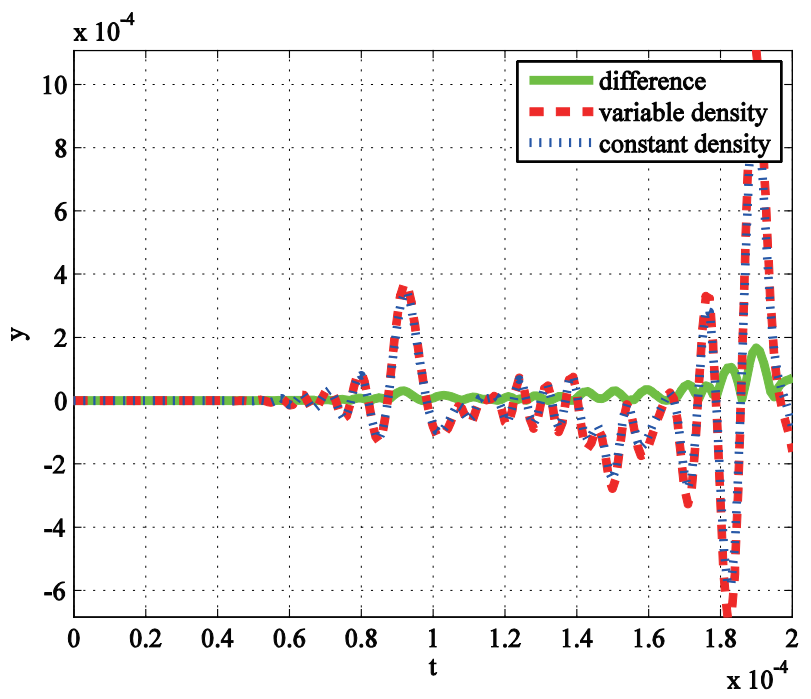

Fig. 5. The potential difference comparison of two kinds of densities tissues and the absolute value of the difference.

The following conclusions can be safely drawn based on the above simulation: (1) when the density is continuously variable, the propagation path of the acoustic wave will change at the interface, but the waveform in the biological tissue is still regular; (2) when the density is continuously variable, the current density and the measured potential difference only differ in amplitude from those measured when the density is constant. In this paper, only the smaller mass density change is considered. In this case, the mass density can be approximately regarded as a constant. For example, when MAET is applied in soft tissue without bones, the mass density can be approximately regarded as a constant.

\section{Discussion}

In many MAET applications, heterogeneous biological tissues are inevitable. Consequently, research on acoustic wave propagation in an inhomogeneous medium is highly significant. In this paper, the equation that the acoustic wave satisfies is given, and its effect on magneto-acoustic-electric tomography is 
addressed when the biological tissue mass density is continuously variable. Furthermore, the acoustic waves were analyzed as well as the impact on magneto-acousto-electrical tomography when the biological tissue mass density was varied. However, there are several other problems worth considering: (1) while the problem of continuous density changes is researched in the present paper, block density changes are not taken into account, and (2) it is still uncertain as to what degree the density can change and yet still be considered approximately constant.

\section{Acknowledgements}

This work was supported by the National Natural Science Foundation of China under Grant Nos. 61427806, 51477161, and 51137004.

\section{References}

[1] Edler I and Lindstrom K, The history of echocardiography, Ultrasound Med Biol. 30 (2004), 1565-1644.

[2] D. Stephens, A. Mahmoud, X. Ding, S. Lucero, D. Dutta, F.T.H. Yu, X. Chen and K. Kim, Flexible integration of high-imaging-resolution and high-power arrays for ultrasound-induced thermal strain imaging, IEEE Trans. Ultrason. Ferroelectr. Freq. Control. 60 (2013), 2645-2656.

[3] Ammari H, Bonnetier E, Capdeboscq Y, Tanter M and Fink M, Electrical impedance tomography by elastic deformation, SIAM J. Appl. Math 68 (2008), 1557-73.

[4] R. Harikumar and R. Prabu, Electrical impedance tomography and its medical applications: a review, International Journal of Soft Computing and Engineering 3 (2013), 193-198.

[5] Woo Chul Jeong, Saurav Z.K. Sajib, Hyung Joong Kim and Oh In Kwon, Focused current density imaging using internal electrode in magnetic resonance electrical impedance tomography, IEEE Trans on Biomedical engineering 61 (2014), 1938-1946.

[6] Leo Mariappan, Xu Li and Bin He, B-Scan based acoustic source reconstruction for magnetoacoustic tomography with magnetic induction, IEEE Trans on Biomedical engineering 58 (2011), 731-720.

[7] Han Wen, Jatin Shah and Robert S. Balaban, Hall Effect Imaging, IEEE Trans on Biomedical engineering 45 (1998), 119-124.

[8] Leonid Kunyansky, A mathematical model and inversion procedure for magneto-acousto-electric tomography, IOP Publishing (2012), Online at stacks.iop.org/IP/28/035002.

[9] Bradley J. Roth and Kevin Schalte, Ultrasonically induced Lorentz force tomography, Med Biol Eng Comput 47 (2009), 573-577.

[10] Tseng N and Roth BJ, The potential induced in anisotropic tissue by the ultrasonically induced Lorentz force, Med Biol Eng Comput 46 (2008), 195-197.

[11] Herman P. Schwan, Electrical and Acoustic Properties of Biological Materials and Biomedical Applications, IEEE Trans on Biomedical Engineering 31 (1984), 872-877. 